\title{
A SHARP VANISHING THEOREM FOR LINE BUNDLES ON K3 OR ENRIQUES SURFACES
}

\author{
ANDREAS LEOPOLD KNUTSEN* AND ANGELO FELICE LOPEZ**
}

\begin{abstract}
Let $L$ be a line bundle on a $\mathrm{K} 3$ or Enriques surface. We give a vanishing theorem for $H^{1}(L)$ that, unlike most vanishing theorems, gives necessary and sufficient geometrical conditions for the vanishing. This result is essential in our study of BrillNoether theory of curves on Enriques surfaces [KL1] and of Enriques-Fano threefolds KLM.
\end{abstract}

\section{INTRODUCTION}

Since Grothendieck's introduction of basic tools such as the cohomology of sheaves and the Grothendieck-Riemann-Roch theorem, vanishing theorems have proved to be essential in many studies in algebraic geometry.

Perhaps the most influential one, at least for line bundles, is the well-known KawamataViehweg vanishing theorem $([\underline{\mathrm{K}}, \mathbf{\mathrm { V }}])$ which, in its simplest form, asserts that $H^{i}\left(K_{X}+\mathcal{L}\right)=0$ for $i>0$ and any big and nef line bundle $\mathcal{L}$ on a smooth variety $X$. On the other hand, as most vanishing theorems (even for special surfaces [CD, Thm.1.5.1]), it gives only sufficient conditions for the vanishing. Practice shows though that, in many situations, it would be very useful to know that a certain vanishing is equivalent to some geometrical/numerical properties of $\mathcal{L}$.

In this short note we accomplish the above goal for line bundles on a K3 or Enriques surface, by proving that, when $L^{2}>0$, the vanishing of $H^{1}(L)$ is equivalent to the fact that the intersection of $L$ with all effective divisors of self-intersection -2 is at least -1 .

In the statement of the theorem we will employ the following

Definition 1.1. Let $X$ be a smooth surface. We will denote by $\sim$ (respectively $\equiv$ ) the linear (respectively numerical) equivalence of divisors (or line bundles) on $X$. We will say that a line bundle $L$ is primitive if $L \equiv k L^{\prime}$ for some line bundle $L^{\prime}$ and some integer $k$ implies $k= \pm 1$.

\section{Theorem.}

Let $X$ be a $K 3$ or an Enriques surface and let $L$ be a line bundle on $X$ such that $L>0$ and $L^{2} \geq 0$. Then $H^{1}(L) \neq 0$ if and only if one of the three following occurs:

(i) $L \sim n E$ for $E>0$ nef and primitive with $E^{2}=0, n \geq 2$ and $h^{1}(L)=n-1$ if $X$ is a $K 3$ surface, $h^{1}(L)=\left\lfloor\frac{n}{2}\right\rfloor$ if $X$ is an Enriques surface;

(ii) $L \sim n E+K_{X}$ for $E>0$ nef and primitive with $E^{2}=0, X$ is an Enriques surface, $n \geq 3$ and $h^{1}(L)=\left\lfloor\frac{n-1}{2}\right\rfloor$;

(iii) there is a divisor $\Delta>0$ such that $\Delta^{2}=-2$ and $\Delta . L \leq-2$.

\footnotetext{
* Research partially supported by a Marie Curie Intra-European Fellowship within the 6th European Community Framework Programme.

** Research partially supported by the MIUR national project "Geometria delle varietà algebriche" COFIN 2002-2004.

2000 Mathematics Subject Classification : Primary 14F17, 14J28. Secondary 14C20.
} 
Note that the hypothesis $L>0$ is not restrictive since, if $L$ is nontrivial, from $L^{2} \geq 0$ we get by Riemann-Roch that either $L>0$ or $K_{X}-L>0$, and $h^{1}(L)=h^{1}\left(K_{X}-L\right)$ by Serre duality.

The theorem has of course many possible applications. For example, if $L$ is base-point free and $|P|$ is an elliptic pencil on $X$, the knowledge of $h^{0}(L-n P)$ for $n \geq 1$ (which follows by Riemann-Roch if we know that $h^{1}(L-n P)=0$ ) determines the type of scroll spanned by the divisors of $|P|$ in $\mathbb{P} H^{0}(L)$ and containing $\varphi_{L}(X)$ ([SD, KJ, Co]). Most importantly for us, this result proves crucial in our study of the Brill-Noether theory [KL1, KL2] and Gaussian maps [KL3] of curves lying on an Enriques surface, and especially in our proof of a genus bound for threefolds having an Enriques surface as a hyperplane section given in KLM].

Acknowledgments. The authors wish to thank Roberto Muñoz for several helpful discussions.

\section{Proof of the Theorem}

We first record the following simple but useful fact.

Lemma 2.1. Let $X$ be a smooth surface and let $A>0$ and $B>0$ be divisors on $X$ such that $A^{2} \geq 0$ and $B^{2} \geq 0$. Then $A . B \geq 0$ with equality if and only if there exists a primitive divisor $F>0$ and integers $a \geq 1, b \geq 1$ such that $F^{2}=0$ and $A \equiv a F, B \equiv b F$.

Proof. The first assertion follows from the signature theorem [BPV, VIII.1]. If $A . B=0$, then we cannot have $A^{2}>0$, otherwise the Hodge index theorem implies the contradiction $B \equiv 0$. Therefore $A^{2}=B^{2}=0$. Now let $H$ be an ample line bundle on $X$ and set $\alpha=A . H, \beta=B$. $H$. We have $(\beta A-\alpha B)^{2}=0$ and $(\beta A-\alpha B) . H=0$, therefore $\beta A \equiv \alpha B$ by the Hodge index theorem. As there is no torsion in $\operatorname{Num}(X)$ we can find a divisor $F$ as claimed.

We now proceed with the theorem.

Proof. One immediately sees that $h^{1}(L)$ has the given values in (i) and (ii). In the case (iii) we first observe that $h^{2}(L-\Delta)=0$. In fact $\left(K_{X}-L+\Delta\right)^{2}>0$, whence if $K_{X}-L+\Delta \geq 0$ the signature theorem [BPV], VIII.1] implies $0 \leq L .\left(K_{X}-L+\Delta\right)=-L^{2}+L . \Delta \leq-2$, a contradiction. Therefore by Riemann-Roch we get

$$
\frac{1}{2} L^{2}+\chi\left(\mathcal{O}_{X}\right)<\frac{1}{2} L^{2}-\Delta . L-1+\chi\left(\mathcal{O}_{X}\right) \leq h^{0}(L-\Delta) \leq h^{0}(L)=\frac{1}{2} L^{2}+\chi\left(\mathcal{O}_{X}\right)+h^{1}(L)
$$

whence $h^{1}(L)>0$.

Now assume that $h^{1}(L)>0$.

First we suppose that $L$ is nef. By Riemann-Roch we have that $L+K_{X}>0$. Since $h^{1}\left(-\left(L+K_{X}\right)\right)=h^{1}(L)>0$, by [BPV], Lemma12.2], we deduce that $L+K_{X}$ is not 1connected, whence that there exist $L^{\prime}>0$ and $L^{\prime \prime}>0$ such that $L+K_{X} \sim L^{\prime}+L^{\prime \prime}$ and $L^{\prime} . L^{\prime \prime} \leq 0$. Now $\left(L^{\prime}\right)^{2} \geq\left(L^{\prime}\right)^{2}+L^{\prime} . L^{\prime \prime}=L^{\prime} . L \geq 0$ and similarly $\left(L^{\prime \prime}\right)^{2} \geq 0$, whence Lemma 2.1 implies that $L^{\prime} \equiv a E, L^{\prime \prime} \equiv b E$ for some $a, b \geq 1$ and for $E>0$ nef and primitive with $E^{2}=0$. This gives us the two cases (i) and (ii).

Now assume that $L$ is not nef, so that the set

$$
\mathcal{A}_{1}(L):=\left\{\Delta>0: \Delta^{2}=-2, \Delta . L \leq-1\right\}
$$

is not empty. Similarly define the set

$$
\mathcal{A}_{2}(L)=\left\{\Delta>0: \Delta^{2}=-2, \Delta . L \leq-2\right\} .
$$


If $\mathcal{A}_{2}(L) \neq \emptyset$ we are done. Assume therefore that $\mathcal{A}_{2}(L)=\emptyset$ and pick $\Gamma \in \mathcal{A}_{1}(L)$. Then $\Gamma . L=-1$, and we can clearly assume that $\Gamma$ is irreducible. Hence if we set $L_{1}=L-\Gamma$ we have that $L_{1}>0, L_{1}^{2}=L^{2}$ and, since $h^{0}\left(L_{1}\right)=h^{0}(L)$, also that $h^{1}\left(L_{1}\right)=h^{1}(L)>0$.

If $L_{1}$ is nef, by what we have just seen, we have $L_{1} \equiv n E$, for $n \geq 2$, whence $L \equiv n E+\Gamma$ and $-1=\Gamma \cdot L=n E . \Gamma-2$, a contradiction.

Therefore $L_{1}$ is not nef and $\mathcal{A}_{1}\left(L_{1}\right) \neq \emptyset$.

If $\mathcal{A}_{2}\left(L_{1}\right) \neq \emptyset$ we pick a $\Delta \in \mathcal{A}_{2}\left(L_{1}\right)$. We have $-2 \geq \Delta . L_{1}=\Delta .(L-\Gamma) \geq-1-\Delta . \Gamma$, whence $\Delta . \Gamma \geq 1,(\Delta+\Gamma)^{2} \geq-2$ and $(\Delta+\Gamma) \cdot L_{1} \leq-1$. Now Lemma 2.1 yields $(\Delta+\Gamma)^{2}=-2$, so that $\Delta . \Gamma=1$. Also $-1 \leq \Delta \cdot L=\Delta \cdot\left(L_{1}+\Gamma\right) \leq-1$, whence $\Delta \cdot L=-1$ and $(\Delta+\Gamma) \cdot L=-2$, contradicting $\mathcal{A}_{2}(L)=\emptyset$.

We have therefore shown that $\mathcal{A}_{2}\left(L_{1}\right)=\emptyset$.

This means that we can continue the process. But the process must eventually stop, since we always remove base components. This gives the desired contradiction.

Remark 2.2. A naive guess, to insure the vanishing of $H^{1}(L)$ for a line bundle $L>0$ with $L^{2} \geq 0$, could be that it is enough to add the hypothesis $L . R \geq-1$ for every irreducible rational curve $R$. However this is not true. Take, for example, a nef divisor $B$ with $B^{2} \geq 4$ and two irreducible rational curves $R_{1}, R_{2}$ such that $B \cdot R_{i}=0, R_{1} \cdot R_{2}=1$. Then $L:=B+R_{1}+R_{2}$ satisfies the above requirements, but $L .\left(R_{1}+R_{2}\right)=-2$, whence $H^{1}(L) \neq 0$ by the theorem.

Remark 2.3. It would be of interest to know if, in the statement of the theorem, it is possible to replace divisors $\Delta>0$ such that $\Delta^{2}=-2$ with chains of irreducible rational curves.

Definition 2.4. An effective line bundle $L$ on a K3 or Enriques surface is said to be quasi-nef if $L^{2} \geq 0$ and $L . \Delta \geq-1$ for every $\Delta$ such that $\Delta>0$ and $\Delta^{2}=-2$.

An immediate consequence of the theorem is

Corollary 2.5. An effective line bundle $L$ on a K3 or Enriques surface is quasi-nef if and only if $L^{2} \geq 0$ and either $h^{1}(L)=0$ or $L \equiv n E$ for some $n \geq 2$ and some primitive and nef divisor $E>0$ with $E^{2}=0$.

\section{REFERENCES}

[BPV] W. Barth, C. Peters, A. van de Ven. Compact complex surfaces. Ergebnisse der Mathematik und ihrer Grenzgebiete 4. Springer-Verlag, Berlin-New York, 1984.

[CD] F. R. Cossec, I. V. Dolgachev. Enriques Surfaces I. Progress in Mathematics 76. Birkhäuser Boston, MA, 1989.

[Co] F. R. Cossec. Projective models of Enriques surfaces. Math. Ann. 265 (1983), 283-334.

[K] Y. Kawamata. A generalization of Kodaira-Ramanujam's vanishing theorem. Math. Ann. 261 (1982), 43-46.

[KJ] T. Johnsen, A. L. Knutsen. K3 projective models in scrolls. Lecture Notes in Mathematics 1842. Springer-Verlag, Berlin, 2004.

[KL1] A. L. Knutsen, A. F. Lopez. Brill-Noether theory of curves on Enriques surfaces I: the positive cone and gonality. Preprint 2006.

[KL2] A. L. Knutsen, A. F. Lopez. Brill-Noether theory of curves on Enriques surfaces II. In preparation.

[KL3] A. L. Knutsen, A. F. Lopez. Surjectivity of Gaussian maps for curves on Enriques surfaces. Adv. Geom. 7 (2007), 215-247.

[KLM] A. L. Knutsen, A. F. Lopez, R. Muñoz. On the extendability of projective surfaces and a genus bound for Enriques-Fano threefolds. Preprint 2006.

[SD] B. Saint-Donat. Projective models of $K-3$ surfaces. Amer. J. Math. 96 (1974), 602-639.

[V] E. Viehweg. Vanishing theorems. J. Reine Angew. Math. 335 (1982), 1-8. 
Andreas Leopold Knutsen, Dipartimento di Matematica, Università di Roma Tre, Largo San Leonardo Murialdo 1, 00146, Roma, Italy. E-MAil knutsen@mat.uniroma3.it

Angelo Felice Lopez, Dipartimento di Matematica, Università di Roma Tre, Largo San Leonardo Murialdo 1, 00146, Roma, ItAly. E-MAIL lopez@mat.uniroma3.it 